\title{
Le alterazioni del metabolismo calcio-fosforo nei pazienti in dialisi: il contributo dello Studio DOPPS. Il punto di vista del Medico
}

\author{
M. Limardo, G. Pontoriero
}

Divisione di Nefrologia, Dialisi e Trapianto, Ospedale A. Manzoni, Lecco

\section{Cos'è il DOPPS?}

Lo studio DOPPS (Dialysis Outcomes and Practice Patterns Study) è uno studio prospettico, osservazionale che ha coinvolto 12 Paesi e si è articolato in quattro fasi successive (1). Il DOPPS I, dal 1996 al 2001, ha visto la partecipazione di oltre 17.000 pazienti di 308 Centri Dialisi di Francia, Germania, Italia, Giappone, Spagna, Gran Bretagna e Stati Uniti. Nel DOPPS II, dal 2002 al 2004, ai precedenti Paesi si sono aggiunti Australia, Nuova Zelanda, Belgio, Canada e Svezia e sono stati coinvolti più di 12.000 pazienti di 322 Centri Dialisi. Al DOPPS III, dal 2005 al 2008, hanno partecipato tutti i Paesi del DOPPS II ed oltre 9.000 pazienti. Il DOPPS IV è attualmente in corso e coprirà il periodo dal 2009 al 2011.

Mediante un sistema di campionamento casuale dei Centri Dialisi e dei pazienti, lo studio DOPPS ha consentito la raccolta di una vasta quantità di dati relativi a caratteristiche demografiche, di comorbilità, di pratica di trattamento e di risultati clinici. L'obiettivo primario dello studio DOPPS è identificare modelli di pratica associati ai migliori risultati clinici nei pazienti affetti da malattia renale cronica (CKD, sigla inglese per Chronic Kidney Disease) in trattamento emodialitico. Tra i principali risultati clinici presi in esame vi sono la mortalità, l'ospedalizzazione, gli accessi vascolari per emodialisi, la qualità di vita. Il DOPPS è senz'altro lo studio osservazionale di più ampio respiro condotto nei pazienti con $\mathrm{CKD}$ in trattamento emodialitico, non solo per il numero di Paesi e di pazienti coinvolti e per la durata nel tempo, ma anche per la molteplicità degli aspetti che sono stati considerati ed analizzati.

\section{DOPPS e metabolismo calcio-fosforo}

Le anomalie del metabolismo calcio-fosforo sono molto comuni nei pazienti affetti da CKD e diversi studi osservazionali hanno evidenziato un'associazione tra queste anomalie e morbilità e mortalità (2-6). Le alterate concentrazioni di calcio, fosforo, paratormone (PTH), fibroblast growth factor-23 (FGF-23) insieme a ridotti livelli di vitamina $\mathrm{D}$ e le alterazioni del turn-over osseo costituiscono una sindrome sistemica che recentemente è stata etichettata con la sigla CKD - MBD (Chronic Kidney Disease - Mineral and Bone Disorder) (7). Per quanto riguarda il metabolismo calcio-fosforo, non abbiamo ancora a disposizione i risultati di studi clinici randomizzati su un ampio numero di pazienti che ci indichino in maniera definitiva quali debbano essere i target terapeutici nei pazienti con $\mathrm{CKD}$, tuttavia abbiamo a disposizione i risultati di studi osservazionali prospettici condotti su un ampio numero di pazienti, come lo studio DOPPS.

I risultati dell'analisi dei dati della fase I e II del DOPPS relativi al metabolismo minerale sono stati pubblicati per la prima volta negli anni 2004 e $2005(9,10)$. Era emerso da questa prima analisi che solo una parte dei pazienti presentava valori di calcio, fosforo e PTH all'interno degli intervalli raccomandati dalle linee guida per il metabolismo minerale allora più diffuse e comunemente accettate a livello internazionale, le KDOQI (Kidney Disease Outcomes Quality Initiative). La percentuale di pazienti in target era del $21.4 \%$ nel DOPPS I, del 26.2\% nel DOPPS II per il PTH, del $40.8 \%$ e $44.4 \%$ per il fosforo e del $40.5 \%$ e $42.5 \%$ per il calcio. Inoltre, lo studio DOPPS aveva confermato l'associazione tra mortalità cardiovascolare e le 


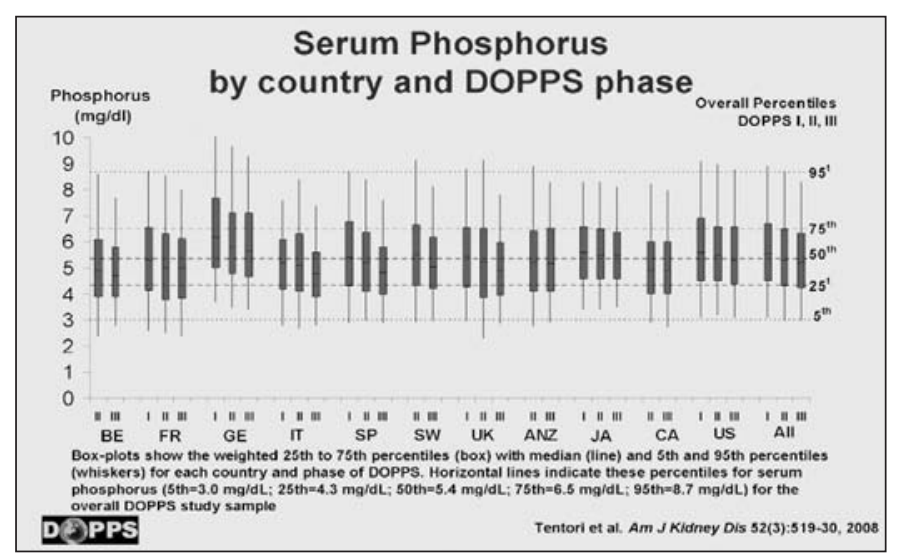

Fig. 1 - Livelli di fosforo nelle fasi I, II e III dello studio DOPPS in ciascun Paese. I riquadri mostrano la distribuzione dal $25^{\circ}$ al $75^{\circ}$ percentile, la linea orizzontale è la mediana, mentre le linee verticali rappresentano il $5^{\circ}$ e il $95^{\circ}$ percentile. Le linee tratteggiate mostrano i valori dei percentili per l'intera popolazione nell'intero periodo (14).

concentrazioni di calcio, fosforo e PTH $(9,10)$.

Nel 2008 sono stati pubblicati da Francesca Tentori et al i risultati DOPPS dell'ultima analisi sulle anomalie del metabolismo calcio-fosforo nei soggetti in emodialisi (11). Questa analisi ha esaminato nel suo insieme le tre prime fasi del DOPPS ed ha valutato una popolazione complessiva di 25.500 pazienti in trattamento emodialitico. Data l'estensione dello studio DOPPS e le modalità di selezione del campione, questi pazienti possono essere considerati come rappresentativi dell'intera popolazione di soggetti in emodialisi a livello mondiale. Questo studio è di particolare interesse per vari motivi. In primo luogo esso dà una descrizione, sul lungo periodo, dei livelli sierici di calcio, fosforo e PTH nei pazienti in dialisi. Poiché questi parametri biochimici rappresentano il target della terapia delle alterazioni del metabolismo calcio-fosforo, il loro andamento esprime anche quanto è stata efficace la terapia messa in atto negli ultimi 10 anni nei pazienti in dialisi. I risultati del lavoro per quanto riguarda questo aspetto sono illustrati sinteticamente nelle Figure 1, 2 e 3 , e mostrano una riduzione dei livelli mediani di calcio e fosforo e una situazione sostanzialmente invariata per quanto riguarda i livelli di PTH. Considerando che c'è stato un miglioramento per quanto riguarda i livelli di calcio e, soprattutto, fosforo, si può dedurre che vi sono stati dei miglioramenti nel trattamento della CKD-MBD negli ultimi 10 anni, probabilmente da attribuire all'implementazione delle linee guida ed alla disponibilità di nuovi farmaci e strategie terapeutiche.

In secondo luogo, questo studio DOPPS analizza il rapporto fra alterazione del metabolismo calcio-fosforo e

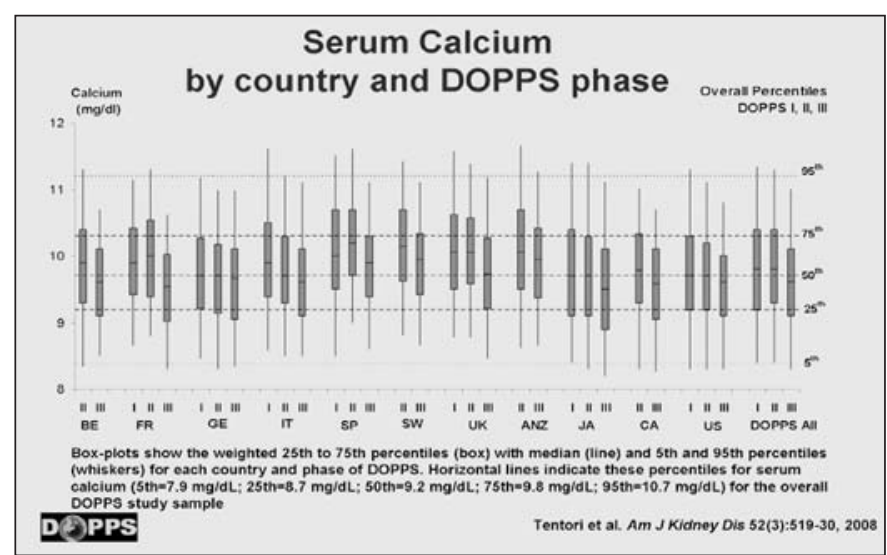

Fig. 2 - Livelli di calcio nelle fasi I, II e III dello studio DOPPS in ciascun Paese (14).

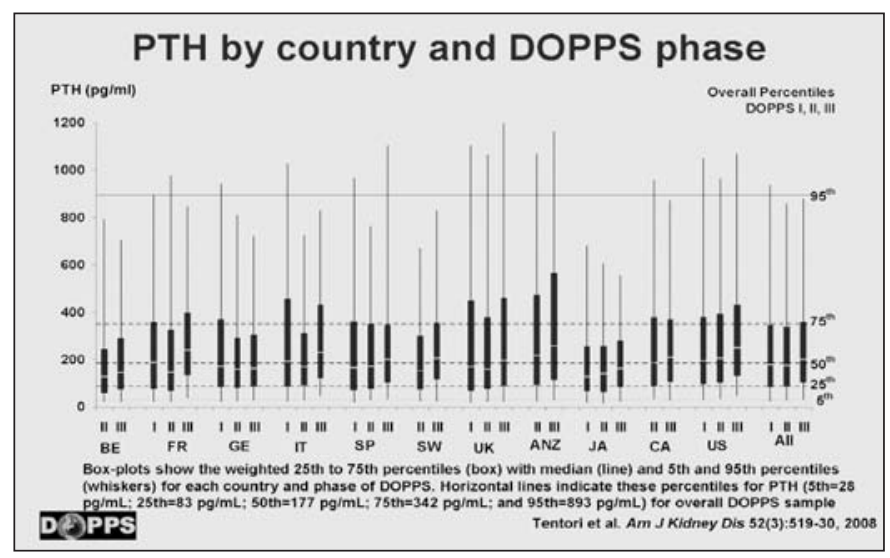

Fig. 3 - Livelli di PTH nelle fasi I, II e III dello studio DOPPS in ciascun Paese (14).

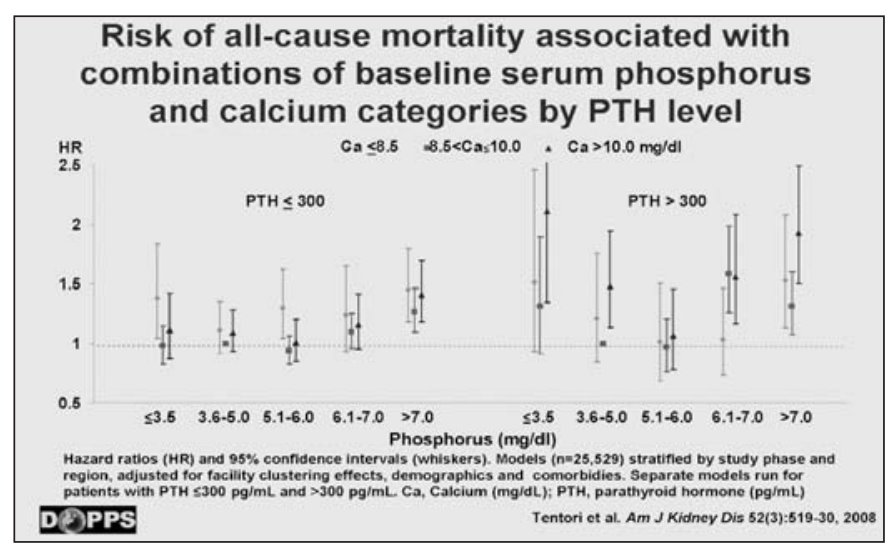

Fig. 4 - Rischio di mortalità da tutte le cause associata con varie combinazioni di intervalli di calcio e fosforo in base ai livelli di PTH $\leq 300$ oppure $>300$ pg/mL (14).

mortalità, generale e cardiovascolare, nei pazienti in dialisi andando ad identificare delle categorie di rischio in base ai livelli di calcio, fosforo e PTH. I risultati di questa 
analisi sono illustrati nella Tabella I. Come si può osservare, livelli di calcio $>10 \mathrm{mg} / \mathrm{dL}(>2.5 \mathrm{mmol} / \mathrm{L})$ sono risultati associati ad una aumentata mortalità. Allo stesso modo, livelli di fosforo $>5.1 \mathrm{mg} / \mathrm{dL}(>1.65 \mathrm{mmol} / \mathrm{L}$ ) sono risultati associati ad una maggiore mortalità cardiovascolare, con un rischio progressivamente maggiore all'aumentare dei livelli di fosforo. Per quanto riguarda il PTH, livelli $>600 \mathrm{pg} / \mathrm{mL}$ sono risultati associati ad una maggiore mortalità per tutte le cause.

Questo studio inoltre sottolinea come sia importante considerare contemporaneamente, e non in modo separato, le alterazioni del metabolismo minerale, perché il rischio cardiovascolare cambia sensibilmente a seconda dell'associarsi delle varie alterazioni del metabolismo minerale. Per esempio, come si può evincere dalla Figura 4, i pazienti con elevati livelli sia di calcio che di fosforo hanno un rischio molto più alto se contemporaneamente hanno anche elevati livelli di PTH. È interessante poi notare che solo i due terzi dei pazienti ad alto rischio presentavano livelli di prodotto calcio $\mathrm{x}$ fosforo $>55 \mathrm{mg}^{2} / \mathrm{dL}^{2}$ $\left(>4.44 \mathrm{mmol}^{2} / \mathrm{L}^{2}\right)$. Questa osservazione riduce il valore del prodotto calcio $\mathrm{x}$ fosforo come indicatore di rischio cardiovascolare e dà maggior importanza alla contem- poranea valutazione dei singoli valori di calcio, fosforo e PTH.

Sulla base di questi risultati gli esperti del DOPPS hanno proposto gli intervalli entro i quali mantenere i livelli sierici di calcio, fosforo e PTH al fine di minimizzare il rischio cardiovascolare dei pazienti in dialisi e questa proposta è stata confrontata con le raccomandazioni delle principali linee guida nazionali ed internazionali (Tab. II). Come messo in evidenza da questa Tabella, vi sono sostanziali differenze tra i target raccomandati dalle diverse linee guida. Emerge dunque la necessità di armonizzare le raccomandazioni delle diverse linee guida integrandole con i dati di più recente pubblicazione come quelli del DOPPS. A questa esigenza ha risposto la recente pubblicazione delle linee guida internazionali KDIGO (Kidney Disease Improving Global Outcomes) per la diagnosi e la terapia della CKD - MBD $(12,13)$. Le linee guida KDIGO, oltre a definire le alterazioni del metabolismo calcio-fosforo, indicano gli obiettivi della terapia e raccomandano o consigliano le strategie terapeutiche da adottare per raggiungerli. Rispetto alle linee guida precedenti, le linee guida KDIGO, anche in accordo con quanto suggerito dai dati DOPPS, sottoli-

\section{Risk for all-cause and cardiovascular mortality associated with DOPPS-derived risk categories for serum calcium, phosphorus and PTH}

\begin{tabular}{|c|c|c|c|c|c|}
\hline \multirow{3}{*}{$\begin{array}{l}\text { Calcium }(\mathrm{mg} / \mathrm{dL}) \text { a } \\
\quad \leq 8.5 \\
8.6-10.0 \\
>10.0\end{array}$} & \multirow{3}{*}{$\begin{array}{c}\mathrm{N}(\%) \\
9,026(25.8) \\
20,621(58.9) \\
5,366(15.3)\end{array}$} & \multicolumn{4}{|c|}{ Hazard Ratio (95\% Confidence Intervals) } \\
\hline & & \multicolumn{2}{|c|}{ All-Cause } & \multicolumn{2}{|c|}{ Cardiovascular } \\
\hline & & $\begin{array}{l}1.02 \\
\text { REF } \\
1.16^{\star \star}\end{array}$ & $\begin{array}{l}(0.94,1.10) \\
(1.08,1.25)\end{array}$ & $\begin{array}{l}0.95 \\
\text { REF } \\
1.24^{\star}\end{array}$ & $\begin{array}{l}(0.83,1.08) \\
(1.10,1.41)\end{array}$ \\
\hline \multicolumn{6}{|c|}{ Phosphorus (mg/dL) ${ }^{b}$} \\
\hline $\begin{array}{l}\leq 3.5 \\
3.6-5.0 \\
5.1-6.0 \\
6.1-7.0 \\
>7.0\end{array}$ & $\begin{array}{c}3,778(10.7) \\
11,041(31.4) \\
8,044(22.9) \\
5,893(16.8) \\
6,411(18.2)\end{array}$ & $\begin{array}{l}1.06 \\
\text { REF } \\
1.02 \\
1.18^{\star} \\
1.43^{\star \star}\end{array}$ & $\begin{array}{l}(0.96,1.16) \\
(0.94,1.09) \\
(1.08,1.28) \\
(1.32,1.56)\end{array}$ & $\begin{array}{l}1.08 \\
\text { REF } \\
1.25^{\star} \\
1.61^{\star \star} \\
1.81^{\star \star}\end{array}$ & $\begin{array}{l}(0.90,1.28) \\
(1.09,1.44) \\
(1.40,1.85) \\
(1.57,2.09)\end{array}$ \\
\hline $\begin{array}{l}\text { PTH }(\mathrm{pg} / \mathrm{mL})^{\mathrm{c}} \\
\leq 100 \\
101-300 \\
301-600 \\
>600\end{array}$ & $\begin{array}{c}7,425(29.0) \\
10,223(39.9) \\
5,038(19.6) \\
2,965(11.6)\end{array}$ & $\begin{array}{l}1.00 \\
\text { REF } \\
1.05 \\
1.21^{\star}\end{array}$ & $\begin{array}{l}(0.95,1.15) \\
(1.08,1.35)\end{array}$ & $\begin{array}{l}1.00 \\
\text { REF } \\
1.05 \\
1.17\end{array}$ & $\begin{array}{l}(0.89,1.23) \\
(0.96,1.43)\end{array}$ \\
\hline
\end{tabular}

PTH, parathyroid hormone, ${ }^{\star} p<0.05 ;{ }^{* \star} p<0.0001$ 


\begin{tabular}{|c|c|c|c|c|}
\hline \multicolumn{5}{|l|}{$\begin{array}{l}\text { Recommende } \\
\text { n Stage } 5 \text { CK }\end{array}$} \\
\hline Country & $\begin{array}{l}\text { Calcium } \\
(\mathrm{mg} / \mathrm{dL})\end{array}$ & $\begin{array}{c}\mathrm{Ca}_{\text {Alb }} \\
(\mathrm{mg} / \mathrm{dL})\end{array}$ & $\begin{array}{l}\text { Phosphorus } \\
\text { (mg/dL) }\end{array}$ & $\begin{array}{c}\text { PTH } \\
(\mathrm{pg} / \mathrm{mL})\end{array}$ \\
\hline ERA-EDTA (2000) & $8.8-11.0$ & -- & $2.4-4.6$ & $85-170$ \\
\hline $\begin{array}{l}\text { UK Renal Association } \\
\text { (2002) }\end{array}$ & -- & $8.8-10.4$ & $<5.6$ & $\begin{array}{l}<4 \text { times } \\
\text { upper normal } \\
\text { range }\end{array}$ \\
\hline $\begin{array}{l}\text { National Kidney } \\
\text { Foundation (2003) }\end{array}$ & --- & $8.4-9.5$ & $3.5-5.5$ & $150-300$ \\
\hline $\begin{array}{l}\text { Canadian Society of } \\
\text { Nephrology (2006) }\end{array}$ & $\begin{array}{l}\text { Within normal } \\
\text { range }\end{array}$ & $\begin{array}{l}\text { Within normal } \\
\text { range }\end{array}$ & $\begin{array}{l}\text { Within normal } \\
\text { range }\end{array}$ & $100-500$ \\
\hline ANZSN (2006) & -- & $8.4-9.5$ & $2.5-5.5$ & $\begin{array}{l}1-3 \text { times } \\
\text { upper normal } \\
\text { range }\end{array}$ \\
\hline $\begin{array}{l}\text { DOPPS-derived lowest } \\
\text { mortality risk category }\end{array}$ & $8.6-10.0$ & $7.6-9.5^{\star}$ & $3.6-5.0^{\prime \prime}$ & $101-600^{\pi}$ \\
\hline
\end{tabular}

neano come a guidare la pratica clinica debba essere la valutazione dei valori di calcio e fosforo e non il costrutto matematico del prodotto calcio $\mathrm{x}$ fosforo. Viene inoltre sottolineato il fatto che deve essere la valutazione dell'andamento nel tempo dei valori di calcio, fosforo e PTH più che i valori presi isolatamente, a guidare la pratica clinica. Le linee guida KDIGO raccomandano di mantenere i livelli di calcio e fosforo dei pazienti in dialisi allinterno degli intervalli di normalità. Per quanto riguarda il PTH, i valori dovrebbero essere mantenuti in un intervallo compreso tra 2 e 9 volte il limite superiore di norma; anche questa raccomandazione è molto vicina a quanto suggerito dallo studio DOPPS.

In sintesi, lo studio DOPPS ha dato un rilevante contributo per la migliore comprensione della patologia renale in uno dei suoi più complessi aspetti, le anomalie del metabolismo minerale e osseo, ed ha così contribuito anche alla stesura e all'aggiornamento di una serie di linee guida nazionali ed internazionali. È auspicabile che i risultati della fase IV del DOPPS, attualmente in corso, consentano di migliorare ulteriormente le nostre conoscenze sul metabolismo minerale ed il suo trattamento perché solo una migliore conoscenza di questo e di altri aspetti del trattamento dialitico ci potrà suggerire le modifiche da apportare alla pratica clinica per migliorare la qualità di vita e ridurre la morbilità e la mortalità dei pazienti in dialisi.

\section{Indirizzo degli Autori:}

Giuseppe Pontoriero, MD

Divisione di Nefrologia, Dialisi e Trapianto

Ospedale A. Manzoni

Via dell'Eremo 9/11

23900 Lecco (LC)

g.pontoriero@ospedale.lecco.it 


\section{Bibliografia}

1. Young EW, Goodkin DA, Mapes DL, et al. The Dialysis Outcomes and Practice Patterns Study (DOPPS): an international hemodialysis study. Kidney Int 2000; 74 (Suppl): S74-S81.

2. Foley RN, Parfrey PS, Harnett JD, et al. Hypocalcaemia, morbidity and mortality in end-stage renal disease. Am J Nephrol 1996; 16: 386-93.

3. Ganesh SK, Stack AG, Levin NW, Hulbert-Shearon T, Port FK. Association of elevated serum PO(4), Ca x PO (4) product and parathyroid hormone with cardiac mortality risk in chronic hemodialysis patients. J Am Soc Nephrol 2001; 12: 2131-8.

4. Block GA, Klassen PS, Lazarus JM, et al. Mineral metabolism, mortality and morbidity in maintenance hemodialysis. J Am Soc Nephrol 2004; 15: 2208-18.

5. Kestenbaum B, Sampson JN, Rudser KD, et al. Serum phosphate levels and mortality risk among people with chronic kidney disease. J Am Soc Nephrol 2005; 16: 520-8.

6. Noordzij M, Korevaar JC, Boeschoten EW, et al. The Kidney Disease Outcomes Quality Initiative (K/DOQI) Guideline for Bone Metabolism and Disease in CKD: association with mortality in dialysis patients. Am J Kidney Dis 2005; 46: 925-32.

7. Kalantar-Zadeh K, Kuwae N, Regidor DL, et al. Survival predictability of time-varying indicators of bone disease in maintenance hemodialysis patients. Kidney Int 2006; 70: $771-80$.
8. Moe S, Drueke T, Cunningham J, et al. Definition, evaluation and classification of renal osteodistrophy: a position statement from Kidney Disease: Improving Global Outcomes (KDIGO). Kidney Int 2006; 69: 1945-53.

9. Young EW, AkibaT, Albert JM, et al. Magnitude and impact of abnormal mineral metabolism in hemodialysis patients in the Dialysis Outcomes and Practice Patterns Study. Am J Kidney Dis 2004: 44 (Suppl 2): S34-S38.

10. Young EW, Albert JM, Satayathum S, et al. Predictors and consequences of altered mineral metabolism: the Dialysis Outcomes and Practice Patterns Study. Kidney Int 2005; 67: 1179-87.

11. Tentori F, Blayney MJ, Albert JM, et al. Mortality risk for dialysis patients with different levels of serum calcium, phosphorus and PTH: the Dialysis Outcomes and Practice Patterns Study (DOPPS). Am J Kidney Dis 2008; 52: 519-30.

12. Kidney Disease: Improving Global Outcomes (KDIGO) CKD-MBD Work Group. KDIGO Clinical Practice Guidelines for the diagnosis, evaluation prevention and treatment of chronic kidney disease-related mineral and bone disorders (CKD-MBD). Kidney Int 2009; 113 (Suppl): S1-S130.

13. KIDIGO home page. URL http://www.kdigo.org/clinical_practice_guidelines/kdigo_guideline_for_ckdmbd.php

14. Welcome to the Dialysis Outcomes and Practice Patterns Study (DOPPS). URL http://www.dopps.org/ 